\title{
FUNDAMENTOS PARA USO SEGURO DAS LAVADORAS TERMODESINFETADORAS COM ÊNFASE NA LIBERAÇÃO PARA USO APÓS INTERVENÇÃO TÉCNICA
}

\author{
Bases for safe use of thermal washer disinfectors emphasizing \\ the release for use after technical intervention
Bases para el uso seguro de las lavadoras termodesinfectantes colocando énfasis en la liberación para el uso después de la intervención técnica

Paulo Roberto Laranjeira', Jeane Aparecida Gonzalez Bronzatti², Rafael Queiroz de Souza ${ }^{3}$, Kazuko Uchikawa Graziano ${ }^{4}$

RESUMO: No cotidiano dos Centros de Material e Esterilização (CMEs), observa-se que as lavadoras termodesinfetadoras submetidas a intervenções técnicas para correção de falhas são liberadas para uso sem evidência de operação conforme os parâmetros requeridos para realizar limpeza e termodesinfecção eficientes. Considerando a importância da prevenção das infecções relacionadas à assistência à saúde, este estudo apresenta como inovação a sistematização dos ensaios requeridos para a liberação de termolavadoras após intervenções técnicas, assim como as informações necessárias para a conservação desses equipamentos em condições ótimas de operação. A liberação segura do equipamento deve incluir a avaliação dos parâmetros de temperatura e tempo em comparação aos dados obtidos na qualificação, a conferência do volume de detergente admitido durante a limpeza, a avaliação da eficácia da limpeza com monitores comercialmente disponíveis, o estabelecimento de um controle de mudanças e um protocolo para direcionar a requalificação, atendendo à legislação nacional e Às recomendações internacionais.

Palavras-chave: Manutenção de equipamento. Detergentes. Desinfecção.

ABSTRACT: It is seen, in the daily routine of Material and Sterilization Centers (CMEs, acronym in Portuguese), that thermal washer disinfectors submitted to technical interventions for correcting failures are released for use without evidence of operation following the required parameters for the effective performance of cleaning and thermal disinfection. Given the importance of preventing healthcare-related infections, this study presents innovated systematization of assays required for the release of thermal washers after technical interventions, as well as the necessary information to preserve such equipment in optimal operation conditions. Safe release of the equipment should include the evaluation of temperature and time parameters compared to data obtained during qualification, the conference of admitted detergent volume during cleaning, the evaluation of cleaning effectiveness with commercially available monitors, the establishment of a change control, and a protocol for directing the requalification, following the Brazilian regulations and international recommendations.

Keywords: Equipment maintenance. Detergents. Disinfection.

'Engenheiro Elétrico. Doutorando da Escola de Enfermagem da Universidade de São Paulo (USP) - São Paulo (SP), Brasil. E-mail: prlaranjeira@usp.br ${ }^{2}$ Enfermeira. Doutoranda da Escola de Enfermagem da USP - São Paulo (SP), Brasil. E-mail: jeanebronzatti@usp.br

${ }^{3}$ Enfermeiro. Mestre e Doutor em Ciências pela Escola de Enfermagem da USP - São Paulo (SP), Brasil. E-mail: rafaelqsouza@hotmail.com

${ }^{4}$ Enfermeira. Professora titular sênior do Departamento de Enfermagem Médico-Cirúrgica da Escola de Enfermagem da USP - São Paulo (SP), Brasil. E-mail: kugrazia@usp.br

Avenida Dr. Enéas de Carvalho Aguiar, 419 - Cerqueira César - CEP: 05403-000 - São Paulo (SP), Brasil.

Recebido: 08 set. 2016 - Aprovado: 15 set. 2016

DOI: $10.5327 / Z 1414-4425201600030009$ 
RESUMEN: En lo cotidiano de los Centros de Material y Esterilización (CMEs), se observa que las lavadoras termodesinfectantes sometidas a intervenciones técnicas para corrección de fallas son liberadas para uso sin evidencia de operación según los parámetros requeridos para realizar limpieza y termodesinfección eficientes. Considerando la importancia de la prevención de las infecciones relacionadas a la asistencia a la salud, este estudio presenta como innovación la sistematización de los ensayos requeridos para la liberación de termolavadoras tras intervenciones técnicas, así como las informaciones necesarias para la conservación de esos equipos en condiciones excelentes de operación. La liberación segura del equipo debe incluir la evaluación de los parámetros de temperatura y tiempo en comparación a los datos obtenidos en la calificación, la conferencia del volumen de detergente admitido durante la limpieza, la evaluación de la eficacia de la limpieza con monitores comercialmente disponibles, el establecimiento de un control de cambios y un protocolo para direccionar la recalificación, atendiendo la legislación nacional y las recomendaciones internacionales.

Palabras clave: Mantenimiento de equipo. Detergentes. Desinfección.

\section{INTRODUÇÃO}

Atualmente, o sistema mundial de atendimento à saúde tem sofrido grande pressão para reduzir custos, aumentar a produtividade e manter a qualidade e segurança, com a necessidade de se manter atualizado perante as novas tecnologias ${ }^{1}$. Com a finalidade de tornar os procedimentos cirúrgicos menos invasivos e traumáticos, o design dos instrumentos tem evoluído consideravelmente, tornando-se cada vez mais complexo, fazendo com que haja a necessidade de um processo de limpeza criterioso, automatizado e reprodutível que traga resultados confiáveis e otimização dos processos de trabalho.

Nesse cenário, as lavadoras termodesinfetadoras têm atendido a essa demanda nos Centros de Material e Esterilização (CMEs). Porém, como todo equipamento, elas exigem qualificação, manutenção e monitoramento para atender aos critérios de segurança e confiabilidade dos resultados ${ }^{2,3}$.

No cotidiano dos CMEs, observa-se que as lavadoras termodesinfetadoras submetidas a intervenções técnicas para correção de falhas são liberadas para uso somente com base nos valores indicados de temperatura e tempo, sem evidência do seu desempenho eficiente na limpeza ou na termodesinfecção. Isso posto, uma vez que os instrumentos que indicam valores de tempo e temperatura no painel do equipamento podem não estar em conformidade com os valores obtidos na qualificação, a limpeza e a termodesinfecção não serão efetivas, comprometendo a segurança no processamento dos produtos para saúde.

A Resolução da Diretoria Colegiada n. ${ }^{\circ} 15$ (RDC-15) da Agência Nacional de Vigilância Sanitária (ANVISA) ${ }^{2}$, assim como a Associação Brasileira de Normas Técnicas $(\mathrm{ABNT})^{3}$, determina a qualificação anual, a calibração, a manutenção, a requalificação e o monitoramento das termolavadoras, para garantir a efetividade dos processos de limpeza e termodesinfecção, no entanto não há instruções sobre os procedimentos que devem ser realizados após uma intervenção técnica.

Levando em conta que a prevenção das infecções relacionadas à assistência à saúde (IRAS), além da segurança do paciente, é tema de relevância global, esta revisão apresenta como inovação a sistematização dos ensaios requeridos para a liberação de termolavadoras após intervenções técnicas, bem como as informações necessárias para a conservação desses equipamentos em condições ótimas de operação.

\section{FASES DO CICLO DE LAVAGEM E TERMODESINFECÇÃ̃O}

De forma geral, a limpeza dos produtos para saúde nas lavadoras termodesinfetadoras ocorre por meio de hastes de pulverização, que utilizam água sob pressão, associadas ao efeito do detergente para auxiliar no desprendimento da sujidade. Para tanto, há suportes específicos (racks) ajustados à conformação dos produtos, que visam promover o alcance da água sob pressão nas superfícies externas e internas.

Os ciclos de limpeza e termodesinfecção realizados nas lavadoras termodesinfetadoras estão apresentados na Figura 1 e descritos com detalhes no texto a seguir:

1. Pré-limpeza: nesta etapa, as superfícies interna e externa dos produtos são expostas a um jato de água fria sob pressão, com a finalidade de remover o excesso de resíduos orgânicos e inorgânicos;

2. Limpeza: realizada com água em temperaturas que, em geral, variam de 40 a $60^{\circ} \mathrm{C}$ pelo período de $5 \mathrm{~min}$, mediante detergente que não produza espuma, de $\mathrm{pH}$ neutro ou alcalino ${ }^{4}$. No Brasil, os detergentes 
enzimáticos, comumente utilizados, devem atender às determinações da RDC-55 da ANVISA, de 20125;

3. Neutralização: esta etapa deve acontecer quando o serviço de saúde optar pelo uso de detergente alcalino. Nesse caso, devem-se adicionar neutralizantes ácidos à água para auxiliar na remoção dos resíduos de detergente e evitar a formação de depósitos de sais";

4. Enxágue: feito com água quente ou fria sem aditivos ${ }^{4}$. De acordo com a RDC-15 da ANVISA, de 2012², esta etapa requer água purificada para o enxágue de produtos críticos empregados em cirurgias de implantes ortopédicos e oftalmológicos, cirurgias cardíacas e neurológicas. Etapas adicionais de enxágue podem ser programadas, por exemplo, para o instrumental oftalmológico e ortopédico;

5. Desinfecção térmica: ocorrida com água purificada em temperaturas que variam entre 80 e $95^{\circ} \mathrm{C}$, com tempo de exposição calculado com base no A0 (A zero) requerido, conforme descrito adiante ${ }^{4}$;

6. Secagem: realizada de forma controlada pela lavadora termodesinfetadora ou por secadoras próprias para produtos para saúde ${ }^{4}$.

O controle da qualidade da água é necessário nas etapas do ciclo. A Association for the Advancement of Medical Instrumentation (AAMI), dos Estados Unidos da América, determina a qualidade de água para o processamento de produtos para saúde e recomenda o controle de bactérias, endotoxinas,

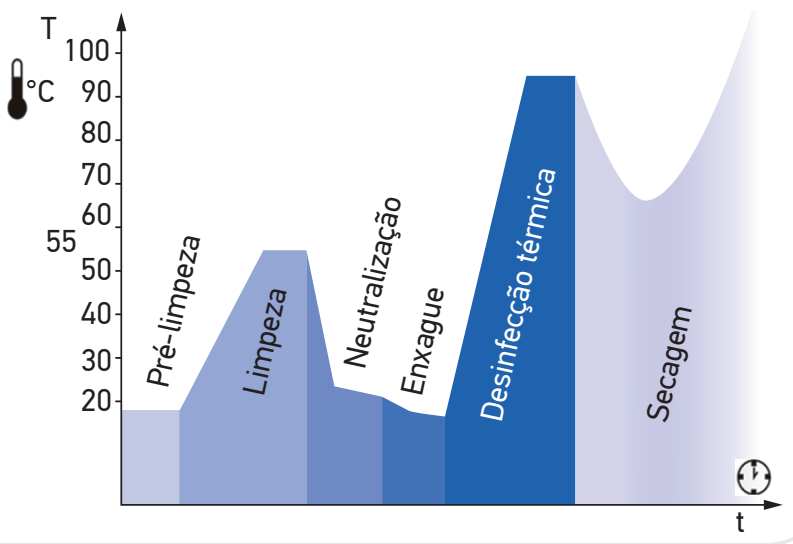

Figura 1. Exemplo das etapas de um ciclo da lavadora termodesinfetadora. Traduzido e adaptado de Arbeitskreis Instrumentenaufbereitung (AKI), $2012^{4}$. carbono orgânico total, $\mathrm{pH}$, dureza, resistividade, sólidos dissolvidos totais, cloreto, ferro, cobre, manganês, cor e turbidez, pois esses fatores podem ocasionar corrosão no instrumental cirúrgico, danificar equipamentos, diminuir a atividade de detergentes e provocar reações tóxicas nos pacientes ${ }^{6}$.

Cabe ao fabricante dos produtos para saúde ou das termodesinfetadoras estabelecer a qualidade da água requerida para o correto funcionamento do equipamento e para a manutenção da qualidade dos produtos, porém essa informação não é claramente difundida e, em alguns casos, ausente.

Uma rotina de monitoramento da água que abastece a lavadora termodesinfetadora deverá ser adotada para medir a pureza química, a temperatura, a pressão de alimentação, a contaminação microbiológica, entre outros, em frequência recomendada pelo fabricante do equipamento ${ }^{3}$. Para que todas as fontes de contaminação sejam monitoradas, sugerese que o ponto de coleta seja o mais próximo possível da entrada da lavadora.

Para a conservação e operação ótima da lavadora termodesinfetadora, os fabricantes apontam os critérios estabelecidos na norma brasileira (NBR) da ABNT ISO 17665-2 $(2013)^{7}$ para a qualidade da água que abastece o equipamento. Os valores estão reproduzidos no Quadro 1.

Alguns componentes da água são de difícil mensuração diária e requerem que amostras sejam enviadas para um laboratório especializado. Há no mercado dispositivos que permitem

Quadro 1. Valores limite recomendados para contaminantes na água de abastecimento de lavadoras termodesinfetadoras, adaptado da NBR ISO 17.665-2:20137.

\begin{tabular}{|l|c|}
\hline Componente & Valor \\
\hline Silicato $\left(\mathrm{SiO}_{2}\right)$ & $\leq 0,1 \mathrm{mg} / \mathrm{L}$ \\
\hline Ferro & $\leq 0,1 \mathrm{mg} / \mathrm{L}$ \\
\hline Cádmio & $\leq 0,005 \mathrm{mg} / \mathrm{L}$ \\
\hline Chumbo & $\leq 0,05 \mathrm{mg} / \mathrm{L}$ \\
\hline $\begin{array}{l}\text { Demais metais pesados, exceto ferro, } \\
\text { cádmio e chumbo }\end{array}$ & $\leq 0,1 \mathrm{mg} / \mathrm{L}$ \\
\hline Cloreto & $\leq 0,1 \mathrm{mg} / \mathrm{L}$ \\
\hline Fosfato $\left(\mathrm{P}_{2} \mathrm{O}_{5}\right)$ & $\leq 0,1 \mathrm{mg} / \mathrm{L}$ \\
\hline Condutividade & $\leq 3 \mu \mathrm{S} / \mathrm{cm}$ \\
\hline pH & $5 \mathrm{a} 7$ \\
\hline Aparência & Limpo, incolor $\mathrm{s}$ \\
\hline Dureza & sem sedimentos \\
\hline
\end{tabular}


medir em tempo real a condutividade da água pelo aumento de inorgânicos dissolvidos, cujos limites operacionais podem ser determinados comparando-se a qualidade da água antes e depois do sistema de tratamento. Assim, quando a condutividade da água ultrapassar o limite estabelecido, sabe-se que a característica físico-química dessa água foi alterada e esta não deverá ser utilizada até a identificação do componente alterado ${ }^{8}$.

Existem ainda testes comercialmente disponíveis que possibilitam monitorar o $\mathrm{pH}$ e a dureza da água (quantidade de $\mathrm{CaCO}_{3}$ e manganês em partes por milhão), por intermédio do uso de fitas que reagem quimicamente com a presença desses componentes, alterando a cor conforme o seu nível, ou sistemas eletrônicos com indicação digital dos valores encontrados ${ }^{8}$.

\section{ROTINAS DE ENSAIOS PARA AVALIAR A OPERAÇÃO DE LAVADORAS TERMODESINFETADORAS}

Os profissionais responsáveis pela operacionalização do CME devem implementar ações que garantam o funcionamento adequado do parque tecnológico do setor, assegurando a qualidade dos produtos processados e evitando interrupções decorrentes de falhas técnicas. As falhas de equipamentos geram atrasos e aumentam os riscos de erros causados por ações emergenciais e planos de contingência. Dessa forma, sugere-se uma rotina de ensaios diários, trimestrais e anuais para avaliação do funcionamento das lavadoras termodesinfetadoras ${ }^{9}$, que tem de ser somada às recomendações do fabricante. Os ensaios de rotina estão apresentados no Quadro 2.

\section{ROTINA PARA LIBERACÃO DE LAVADORAS TERMODESINFETADORAS APÓS INTERVENÇÃO TÉCNICA}

A liberação segura de lavadoras termodesinfetadoras após a intervenção inclui não somente a avaliação da temperatura e do tempo do ciclo, mas também a conferência do volume de detergente admitido durante a limpeza e a avaliação da eficácia da limpeza com monitores comercialmente disponíveis, em associação às diretrizes para elaboração

Quadro 2. Diretrizes para elaboração de um plano de avaliação e manutenção da operação de lavadoras termodesinfetadoras, de acordo com a periodicidade.

\begin{tabular}{|c|c|c|c|}
\hline \multirow{2}{*}{ Item a ser avaliado } & \multicolumn{3}{|c|}{ Periodicidade } \\
\hline & Diária & Trimestral & Anual \\
\hline Condutividade da água** & $x$ & & \\
\hline Volume de detergente & $x$ & & \\
\hline Limpeza e fixação da grelha do reservatório de água dentro da câmara & $X$ & & \\
\hline Movimento das hastes de pulverização & $X$ & & \\
\hline Papel da impressora & $x$ & & \\
\hline Cartucho de tinta da impressora & $x$ & & \\
\hline Vazamentos de água & $\mathrm{X}$ & & \\
\hline Inspeção visual da limpeza de todas as cargas & $\mathrm{X}$ & & \\
\hline Indicação de baixo volume de detergente* & & $\mathrm{X}$ & \\
\hline Sistema de dosagem do volume de detergente* & & $\mathrm{X}$ & \\
\hline Eficácia da limpeza utilizando simuladores de sujidade* & & $X$ & \\
\hline Temperatura de um ciclo com carga, com instrumento externo* & & $x$ & \\
\hline Sistema de travamento das portas* & & $x$ & \\
\hline Qualificação (no mínimo)** & & & $x$ \\
\hline Calibração (no mínimo) ${ }^{\star *}$ & & & $x$ \\
\hline Requalificação & \multicolumn{3}{|c|}{$\begin{array}{l}\text { De acordo com os eventos estabelecidos } \\
\text { no controle de mudanças }\end{array}$} \\
\hline
\end{tabular}

*NBR ISO 15.883-13; **RDC-15 da ANVISA, de 2012². 
do plano de avaliação e manutenção da operação, descritas anteriormente.

Para verificar se a diluição do detergente se mantém de acordo com a quantidade programada, deve-se utilizar uma proveta graduada. Esta deve ser posicionada na saída do dosador dentro da lavadora. Em seguida, aciona-se o comando de dosagem; o volume obtido dentro da proveta representa a quantidade de produto admitido em cada ciclo. O resultado dessa ação deve ser comparado aos parâmetros preestabelecidos para o equipamento e às recomendações do fabricante do detergente, comprovando, assim, se o volume de detergente admitido em cada ciclo de lavagem está correto.

A seguir, é necessário programar um ciclo de limpeza, excluindo-se as fases de termodesinfecção e secagem, desafiando a limpeza com testes que simulem sujidade, conforme recomendações da ISO 15.883-5:2005 ${ }^{10}$. Para realização do monitoramento da limpeza, estão disponíveis diversos monitores com diferentes marcadores, como proteínas, sangue e adenosina trifosfato (ATP $)^{10-13}$. A interpretação dos resultados obtidos com esses produtos precisa ser realizada de acordo com as orientações do fabricante, entretanto ressalta-se que não há testes rápidos para detecção de resíduos de gordura, biofilmes e proteínas priônicas para uso na rotina do CME.

Uma vez que o equipamento é aprovado na fase de limpeza, deve-se verificar se a termodesinfecção está sendo alcançada, com um indicador comercialmente disponível em um ciclo padrão, que demonstrará se o tempo e a temperatura programados foram de fato atingidos ${ }^{3}$. Os parâmetros de tempo de exposição e temperatura são desafiados durante o processo de qualificação térmica, a qual tem de ocorrer no mínimo anualmente ${ }^{2}$, em conformidade com a frequência e os procedimentos apontados pelo fabricante ou caso algum componente dessa cadeia de medição tenha sido substituído.

A fase de termodesinfecção tem a sua eficiência determinada por meio do cálculo estatístico, identificado por A0 (A zero). Esse conceito está definido na ISO 15.883-1:2013³, na qual, com base no processo de desinfecção, durante um tempo específico a dada temperatura, acontece letalidade previsível sobre uma população de microrganismos padronizados.

A fórmula utilizada para calcular o A0, apresentada na Figura 2, é exatamente igual à fórmula usada para calcular o F0 (F zero) nos processos de esterilização pelo vapor saturado, em que $\mathrm{F}$ indica a temperatura referência de $121^{\circ} \mathrm{C}$, e, no caso da termodesinfecção, o A indica a temperatura referência de $80^{\circ} \mathrm{C}$. O valor $Z$ representa a variação da temperatura que vai determinar a redução de $1 \log$ para cada instante e é fixado em $10^{\circ} \mathrm{C}$. Portanto, a fórmula fornecerá o tempo total requerido para se obter o nível de desinfecção desejado.

Essa fórmula foi concebida com a temperatura de referência de $80^{\circ} \mathrm{C}$, pressupondo que a elevação dessa temperatura diminuiria o tempo de exposição, e não o contrário. Isso posto, não é recomendada a utilização dessa fórmula para temperaturas abaixo de $80^{\circ} \mathrm{C}^{3}$. Para estas, a fórmula de A0 só pode ser utilizada até o limite de $70^{\circ} \mathrm{C}$, sendo sugerido no mínimo $75^{\circ} \mathrm{C}$, para que a inativação de bactérias e vírus termorresistentes ocorram em equivalência com a integração do $\mathrm{A} 0^{14}$. Como a inativação de bactérias como Enterococcus, Legionella e alguns tipos de protozoários é geralmente efetiva a temperaturas acima de $65^{\circ} \mathrm{C}$, a integração da fórmula do A0 só deve ser realizada com essa temperatura em diante ${ }^{14}$.

$O$ valor de A0 é calculado em segundos. A norma NBR ISO $15.883-1^{3}$ determina que o valor mínimo deve ser de 600 segundos para produtos que serão esterilizados e 3.000 segundos para os que não serão esterilizados ${ }^{15}$. Os tempos de exposição para cada temperatura estão no Quadro 3.

Não há disponível no mercado nacional indicador que possa ser utilizado como referência para a determinação

$$
A 0=\sum 10^{\frac{(T-T r e f)}{Z}} \times \Delta t
$$

Fonte: ABNT NBR ISO 15883-1:2013

Figura 2. Fórmula do $\mathrm{AO}$.

Quadro 3. Tempo mínimo de exposição para cada temperatura para o AO requerido, conforme NBR ISO $15.883-1: 2013^{3} \mathrm{e}$ Rosenberg (2003) $)^{15}$.

\begin{tabular}{|l|c|c|}
\hline A0 (segundos) & Temperatura $\left(^{\circ} \mathbf{C}\right)$ & Tempo (minutos) \\
\hline \multirow{3}{*}{600} & 80 & 10 \\
& 90 & 1 \\
\hline \multirow{3}{*}{3.000} & 93 & 0,5 \\
& 80 & 50 \\
\hline
\end{tabular}


do A0 nem como dispositivo de monitoramento da fase de desinfecção térmica com comprovação de redução de carga microbiana. Os únicos dispositivos comerciais disponíveis são os indicadores químicos que informam se a temperatura foi alcançada por um determinado tempo de exposição.

Parâmetros para termodesinfecção baseados em A0, de acordo com as referências de Rosenberg ${ }^{15}$ e da $\mathrm{ABNT}^{3}$, são ainda de difícil compreensão e implementação por parte dos CMEs brasileiros por várias razões, inclusive pela termossensibilidade dos produtos.

Na termodesinfecção não é recomendada a fórmula para cálculo do $\mathrm{A} 0$ para temperaturas abaixo de $80^{\circ} \mathrm{C}$. Caso o cálculo fosse para $70^{\circ} \mathrm{C}$ (temperatura frequentemente adotada em nosso meio para termodesinfecção de produtos para inaloterapia e assistência ventilatória), o tempo requerido, segundo a fórmula, seria 500 minutos a $70^{\circ} \mathrm{C}$ para $\mathrm{A} 0$ de 3.000 e 100 minutos para A0 de 600 .

$\mathrm{O}$ vírus da hepatite $\mathrm{B}$ requer 20 minutos a $80^{\circ} \mathrm{C}$ para ser inativado, e para a temperatura de $70^{\circ} \mathrm{C}$, seriam necessários quase 200 minutos para alcançar a mesma redução ${ }^{16}$.

Em 2000, uma investigação avaliou a eficácia dos parâmetros de $77^{\circ} \mathrm{C}$ por 30 minutos empregados por uma pasteurizadora (um "banho-maria" com controle de temperatura) para desinfecção de dispositivos de assistência ventilatória e de anestesia. Um alto número de microrganismos teste ( $10^{4}$ a $10^{6}$ unidades formadoras de colônia) foi inoculado em tubos plásticos e metálicos ( $3 \mathrm{~mm}$ de diâmetro e $40 \mathrm{~cm}$ de comprimento) e submetido a um ciclo de termodesinfecção. Foram eliminados Staphylococcus aureus, Klebsiella pneumoniae, Candida albicans e Mycobacterium terrae, porém os esporos do Bacillus subtilis não foram eliminados. Nesse estudo, os autores concluíram que o equipamento é eficiente para dispositivos de assistência ventilatória e de anestesia e enfocaram a pasteurização como uma alternativa para a desinfecção utilizando desinfetantes químicos ${ }^{17}$. Portanto, a liberação e o monitoramento da fase de termodesinfecção para temperaturas abaixo de $80^{\circ} \mathrm{C}$, com base na integração do A0, devem possuir sustentação teórica e científica que comprovem que o tempo e a temperatura adotados sejam adequados para alcançar a eficácia requerida para o processo.

Como todo equipamento é passível de falha, recomendase elaborar um protocolo de avaliação de mudanças, estabelecendo o impacto das intervenções na performance do equipamento, estipulando as etapas de qualificação que devem ser repetidas a fim de determinar a continuidade de operação na faixa de trabalho previamente desafiada na qualificação anual. Para auxiliar na montagem de um protocolo, sugere-se consulta à NBR ISO 17.665-1 ${ }^{18}$, da ABNT, que fornece diretrizes para a validação do processo de esterilização e que pode ser adaptada para a termodesinfecção. Esse documento, assim como o presente trabalho, deve ajudar os profissionais que atuam em CME a determinar quando o equipamento precisa ser requalificado após a intervenção técnica, atendendo às exigências do artigo 41 da RDC-15 da ANVISA, de $2012^{2}$.

Uma vez que a NBR ISO $17.665-1^{18}$ se destina aos equipamentos de esterilização pelo vapor, recomenda-se a construção de uma norma específica para a elaboração de um protocolo de avaliação de mudanças, com as etapas da qualificação que requerem a repetição após intervenções técnicas em lavadoras termodesinfetadoras. Os resultados obtidos com os procedimentos têm de ser confrontados com os testes realizados durante a qualificação, incluindo a comparação com os registros físicos, e, se estiverem em conformidade, a lavadora termodesinfetadora poderá ser liberada para uso.

\section{CONCLUSÃO}

A adoção de um procedimento contínuo de ensaios para avaliar a operação das lavadoras termodesinfetadoras permite diagnosticar precocemente falhas, trazendo mais controle e qualidade ao processo automatizado de limpeza e termodesinfecção.

Os equipamentos submetidos à intervenção merecem atenção antes da sua utilização na rotina, pois somente os dados fornecidos no painel não atestam a segurança do processo de limpeza e termodesinfecção. A liberação segura do equipamento deve incluir a avaliação dos parâmetros de temperatura e tempo em comparação aos dados obtidos na qualificação, na conferência do volume de detergente admitido durante a limpeza, na avaliação da eficácia da limpeza com monitores comercialmente disponíveis e no estabelecimento de um controle de mudanças e de um protocolo para direcionar a requalificação, atendendo à legislação nacional e às recomendações internacionais. 


\section{REFERÊNCIAS}

1. Giambrone GP, Hemmings HC, Sturm M, Fleischut PM. Information technology innovation: the power and perils of big data. $\mathrm{Br} \mathrm{J}$ Anaesth. 2015;115(3):339-42.

2. Brasil. Ministério da Saúde. Resolução da Diretoria Colegiada nº 15 , de 15 de março de 2012. Dispõe sobre requisitos de boas práticas para o processamento de produtos para saúde e dá outras providências. Brasília: 2012.

3. Associação Brasileira de Normas Técnicas (ABNT). NBR ISO 15883, Lavadoras desinfetadoras. Parte 1: Requisitos gerais, termos, definições e ensaios. Rio de Janeiro: ABNT; 2013.

4. Arbeitskreis Instrumenten-Aufbereitung. Instrument reprocessing: reprocessing of instruments to retain value, 10. Ed. 2012. Disponivel em: http://www.a-k-i.org/index.php?id=11\&L=1

5. Brasil. Ministério da Saúde. Resolução da diretoria colegiada $n^{\circ}$. 55, de 14 de novembro de 2012. Dispõe sobre os detergentes enzimáticos de uso restrito em estabelecimentos de assistência à saúde com indicação para limpeza de dispositivos médicos e dá outras providências. Brasília: 2012.

6. Association for the Advancement of Medical Instrumentation (AAMI). Water for the reprocessing of medical devices. Arlington, VA: Association for the Advancement of Medical Instrumentation; 2007. AAMI Technical information report 34. TIR34:2007.

7. Associação Brasileira de Normas Técnicas (ABNT). NBR ISO 176652, Esterilização de produtos para saúde - Vapor - Parte 2: Guia de aplicação da ABNT NBR ISO 17665-1. Rio de Janeiro: ABNT; 2013.

8. Blechschmidt T. Routine Control of Washer-Disinfectors. Zentralsterilisation - Central Service. 2012;5:330-5.

9. Harty Z, Benz L. Washer monitoring: A must-have for today's best practices (part 2). Healthcare Purchasing News. Self-Study Series. Sarasota (FL): KSR Publishing; 2012. p. 30.
10. International Standard Organization. ISO 15883-5, Washerdisinfectors. Part 5: Test soils and methods for demonstrating cleaning efficacy; 2005.

11. Alfa MJ, Olson N. Comparison of washer-disinfector cleaning indicators: impact of temperature and cleaning cycle parameters. Am J Infect Control. 2014;42(2):e23-6.

12. Alfa MJ. Monitoring and improving the effectiveness of cleaning medical and surgical devices. Am J Infect Control. 2013;41(5 Suppl):S56-9.

13. Alfa MJ, Olson N, Al-Fadhaly A. Cleaning efficacy of medical device washers in North American healthcare facilities J Hosp Infect. 2010;74(2):168-77.

14. McCormich PJ, Schoene MJ, Dehmler MA, McDonnell G. Moist heat disinfection and revisiting the $\mathrm{AO}$ Concept. Biomedical Instrumentation \& Technology: Challenges and Solutions for Medical Devices; 2016. p. 19

15. Rosenberg, U. Thermal Disinfection - The A0 Concept and the Biological Background. Zentralsterilisation - Central Service. 2003;11:118-20.

16. Uetera Y, Kawamura K, Kobayashi H, Saito Y, Yasuhara H, Saito R. Studies on Viral Disinfection: An evaluation of moist heat disinfection for HBV by using AO concept defined in ISO 15883-washer-disinfectors. PDA J Pharm Sci Technol. 2010;64(4):327-36.

17. Rutala WA, Weber JD, Gergen MF, Gratta A R. Efficacy of a washerpasteurizer for disinfection of respiratory-care equipment. Infect Control Hosp Epidemiol. 2000;21(5):333-6.

18. Associação Brasileira de Normas Técnicas (ABNT). NBR ISO 17665-1, Esterilização de produtos para saúde - Vapor - Parte 1: Requisitos para o desenvolvimento, validação e controle de rotina nos processos de esterilização de produtos para saúde. Rio de Janeiro: ABNT; 2010. 